(C) 1984. The Genetical Society of Great Britain

\title{
INTERACTION BETWEEN DIFFERENT GENOTYPES OF ALLOGAMOUS AND AUTOGAMOUS RYE AND THE HOMOEOLOGOUS PAIRING CONTROL OF WHEAT
}

\author{
M. C. CUADRADO AND C. ROMERO \\ Departamento de Genetica, Facultad de Biologia, Universidad Complutense, Madrid 3, Spain \\ Received 13.vi.83
}

\section{SUMMARY}

In this study, we have analysed meiotic pairing in wheat-rye intergeneric hybrids obtained by crossing $T$. aestivum cv. Chinese Spring with two cultivars of $S$. cereale (Elbon and Ailés, respectively) and $S$, vavilovii.

The results have shown that meiotic behaviour of hybrids is different in relation to the type of rye utilised. This has been interpreted as being due to the different effect of each particular rye genotype on the homoeologous pairing in relation with the reproductive systems (allogamy or autogamy) of the particular rye.

\section{INTRODUCTION}

Wheat has a mechanism to maintain its meiotic regularity which is effective even in single doses. The studies made using hybrids between $T$. aestivum and other related species have shown that the pairing level is low and with values close to these observed in wheat haploids, although there are some reports of particular genotypes in which the homoeologous pairing is promoted, namely in $T$. speltoides (Dvorak, 1972), $T$. tripsacoides (Dover and Riley, 1972) and T. longissimum (Mello-Sampayo, 1971).

$S$. cereale genome in its hybrids with $T$. aestivum has no clear effect on homoeologous pairing. Upadhya and Swaminathan (1967) have suggested that the mere fact of being hybrid could promote pairing. Other investigators have indicated that several doses of $\mathbf{R}$ genome (rye) are necessary to detect an increase in hybrid homoeologous pairing (Miller and Riley, 1972; Naranjo et al., 1979). However, there is evidence about the existence of genetic combinations in rye which are able to promote hybrid homoeologous pairing (Wall et al., 1971; Roothan and Sybenga, 1976; Dvorak, 1977). On the other hand, the data given by Lelley (1976) and Romero and Lacadena (1982) suggest the possible existence in rye of promotor and supressor genes for homoeologous pairing.

In this study we have analysed the effect of different genetic combinations of autogamous and allogamous rye on the wheat homoeologous pairing regulation systems using intergeneric hybrids between hexaploid wheat and diploid rye.

\section{MATERIALS AND METHODS}

Hybrids obtained by crossing $T$. aestivum L. cv. Chinese Spring as female with $S$. vavilovii (HCSV), $S$. cereale cv. Elbon (HSCE), and $S$. cereale cv. Ailés (HSCA) as males, were used in the present research. The hybrid seeds were allowed to grow in culture medium (Kaltsikes, 1974) then transplanted into pots and grown outdoors to maturity. The meiotic analysis was made 
on Metaphase I anthers stained by the Feulgen method. The slides were prepared by the acetocarmine squash technique and made permanent with Sandeural. 50 pollen mother cells (PMCs) were scored for each plant from the most synchronus Metaphase I slide, with the exception of 3 plants in which it was only possible to score 30,25 , and 11 PMCs. The chiasmata number was estimated as the number of bound arms. The paired chromosome complement was estimated as the number of paired chromosomes in relation to the total number of chromosomes. The comparisons between bivalent, chiasmata per cell and paired chromosome complement distributions were made by the $\chi^{2}$ contingency test, and the comparison between the variance distributions by the $F$ test.

\section{Results}

55 wheat-rye hybrids $(2 n=28)$ were analysed. The data of 27 previously obtained $T$. aestivum cv. Chinese Spring $\times S$. cereale cv. JNK (HCSJ) hybrids were also used (Romero and Lacadena, 1982).

Table 1 is a summary of the meiotic pairing values of the HCSE, HCSA, and HCSV hybrids. For HCSE plants the mean value of bivalents per cell was 0.27 , ranging from 0.00 to 1.08 , and the chiasmata per cell mean coincided in most of the plants with the bivalent mean. In some cases, these parameters had different values and this was due to the ring bivalent and multivalent association occurrence, mainly trivalents and quadrivalent in one case. These plants had a low paired chromosome complement with a mean value of 0.02 .

In the HCSA hybrids, the mean value of bivalent per cell was 0.44 , ranging from 0.08 to 2.56 . The meiotic behaviour of HCSA plants was similar to that of HCSE hybrids and the bivalent mean coincided in almost all cases with chiasmata per cell mean. The paired chromosome complement was $0 \cdot 03$, slightly higher than the HCSE value. The comparison by $\chi^{2}$ contingency test for the distribution of bivalents and chiasmata per cell did not show significant differences either in HCSE or in HCSA plants.

The mean value of bivalents per cell shown for HSCV plants was 0.44 ranging from 0.34 to 0.54 . In this case, the mean of chiasmata per cell coincided with the mean value of bivalents. The paired chromosome complement was $0 \cdot 03$.

Table 2 shows the comparison by $\chi^{2}$ contingency test of the bivalents and paired chromosome complement distributions of the hybrids taken two by two. For HSCE and HCSA hybrids the $\chi^{2}$ contingency test indicated significant differences both for bivalents and paired chromosome complement distributions. The same result was obtained in HCSE-HCSJ and HCSA-HCSJ comparisons. In the case of the comparison of HCSE-HCSV bivalent distribution, the $\chi^{2}$ did not indicate significant differences. However, $\chi^{2}$ indicated highly significant differences in the paired chromosome complement distribution. The two distributions were not significant for HCSAHCSV and HCSJ-HCSV comparisons.

The variances of bivalent and paired chromosome complement distribution were calculated for HCSE, HCSA, HCSJ and HCSV hybrids. These results are summarised in table 3(a). The HCSA hybrids have the highest variance values in both bivalents and paired chromosome complement $(0.3790$ and 0.0025 , respectively). The HCSV hybrids have the lowest 


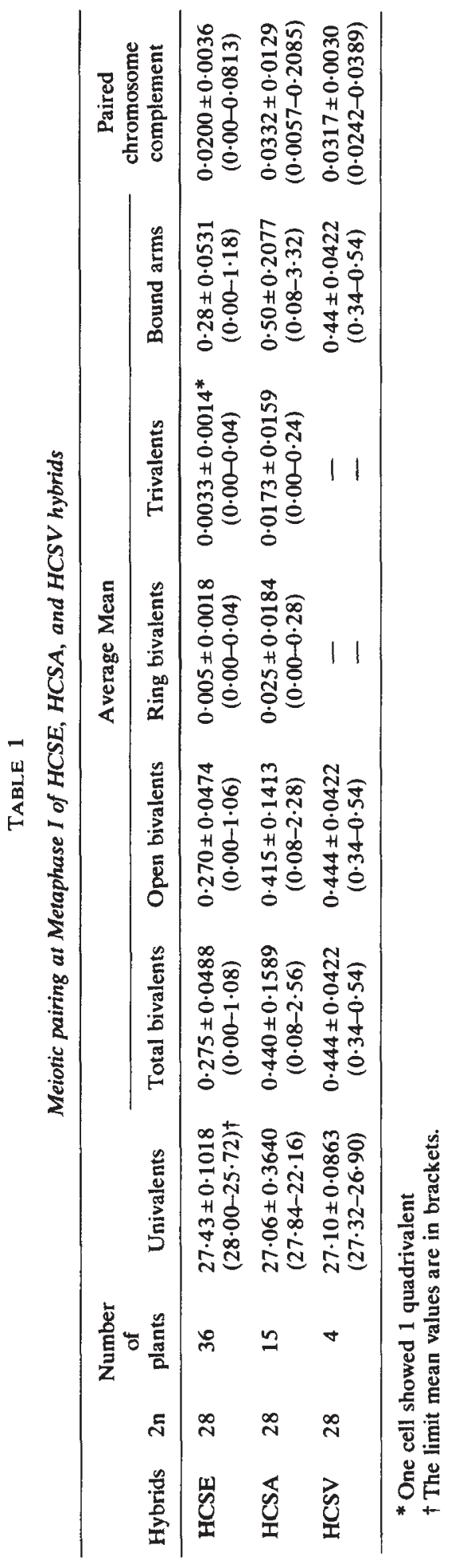


TABLE 2

The comparison of bivalent and paired chromosome complement between the different hybrids take two by two

\begin{tabular}{lcccccc}
\hline $\begin{array}{c}\text { Compared } \\
\text { hybrids }\end{array}$ & $\begin{array}{c}\chi^{2} \\
\text { bivalent }\end{array}$ & df & $\begin{array}{c}\text { significance } \\
\text { level }\end{array}$ & $\begin{array}{c}\chi^{2} \\
\text { paired chr. } \\
\text { complem. }\end{array}$ & df & $\begin{array}{c}\text { significance } \\
\text { level }\end{array}$ \\
\hline HCSE-HCSA & 17.29 & 3 & $\mathrm{P}<0.001$ & 33.87 & 1 & $\mathrm{P}<0.001$ \\
HCSE-HCSJ & 51.26 & 3 & $\mathrm{P}<0.001$ & 106.37 & 1 & $\mathrm{P}<0.001$ \\
HCSA-HCSJ & 18.46 & 4 & $\mathrm{P}<0.001$ & 6.58 & 1 & $0.01<\mathrm{P}<0.02$ \\
HCSE-HCSV & 4.58 & 2 & $0.10<\mathrm{P}<0.20$ & 17.13 & 1 & $\mathrm{P}<0.001$ \\
HCSA-HCSV & 0.80 & 2 & $0.50<\mathrm{P}<0.75$ & 0.86 & 1 & $0.25<\mathrm{P}<0.50$ \\
HCSJ-HCSV & 1.79 & 2 & $0.25<\mathrm{P}<0.50$ & 0.15 & 1 & $0.50<\mathrm{P}<0.75$ \\
\hline
\end{tabular}

TABLE 3

The variance values of bivalent and paired chromosome complement distributions of HCSE, HCSA, HCSJ and HCSV hybrids (a). Grouped variance of HCSE, HCSA, HCSJ respect to HCSV hybrids (b)

(a)

\begin{tabular}{cc}
$V_{\text {HCSE }}^{\text {bivalent }}=0.0860$ & $V_{\text {H.C.c. }}^{\text {p.c.c. }}=0.0004$ \\
$V_{\text {HCSA }}^{\text {bivalent }}=0.3790$ & $V_{\text {H.c.c. } A}^{\text {p.c. }}=0.0025$ \\
$V_{\text {HCS } J}^{\text {bivlalent }}=0.0732$ & $V_{\text {H.c.c. }}^{\text {. }}=0.0003$ \\
$V_{\text {HCSV }}^{\text {bivalent }}=0.0071$ & $V_{\text {HCS.c. }}^{\text {p.c. }}=0.00003$ \\
p.c.c. $=$ paired chromosome complement & \\
\hline
\end{tabular}

(b)

\begin{tabular}{|c|c|c|}
\hline $\begin{aligned} V_{\text {HCSE,HCSA,HCSJ }}^{\text {bivalent }} & =0.1362 \\
V_{\text {HCSV }}^{\text {bivalent }} & =0.0071\end{aligned}$ & $\mathrm{~F}=19 \cdot 19$ & $0.01<\mathrm{P}<0.05$ \\
\hline VF.c.c. ${ }_{\text {CSE,HCSA,HCSJ }}=0.0008$ & & \\
\hline$V_{\text {HCSV }}^{\text {p.c.c. }}=0.00003$ & $F=27 \cdot 55$ & $\mathrm{P}<0.001$ \\
\hline
\end{tabular}

$(0.0071$ and 0.00003 , respectively). The average mean of different hybrid types has not been compared by the " $t$ " test because there was a large range of variation in the distributions.

The pairing data in four hybrid types is shown in fig. 1. The points indicate the bivalent mean for each plant and the crosses the mean value of each hybrid type. In this figure, the plants that are homogeneous are indicated by a line. When it was not possible to carry out the homogeneity $\chi^{2}$ test for different reasons (classes with expected values lower than five; small number of cells in the classes with at least one bivalent or large differences between pairing levels), the plants are not grouped.

In figure 1, a large variation of pairing in each hybrid type and between different hybrids can be observed. The HSCV plants have the lowest variation range.

The pairing levels have been divided into five groups according to an arbitrary nomenclature; the plants located in 0.00 to 0.04 are included in 


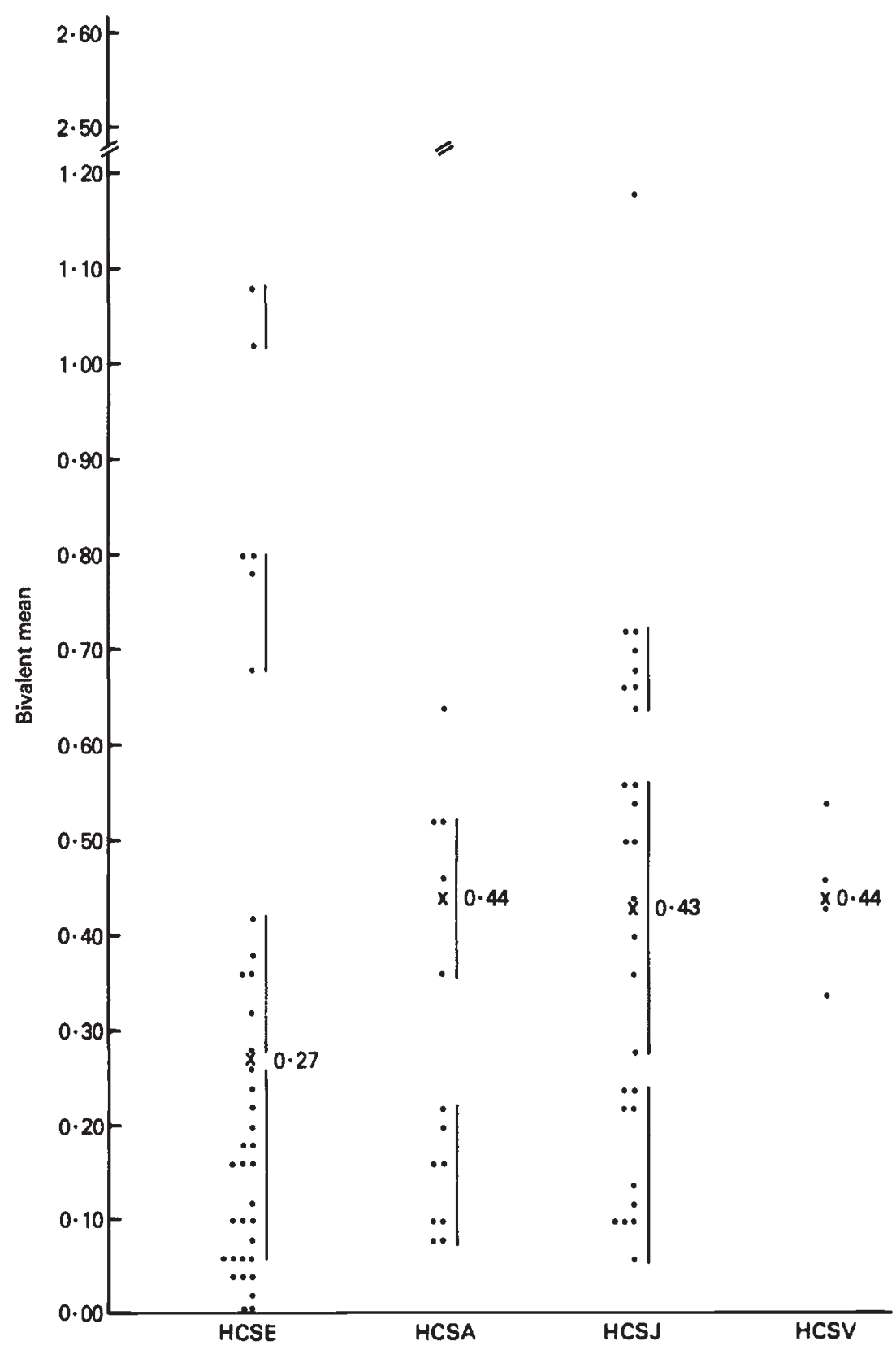

FIG. 1

the group named "low pairing". The values from 0.06 to 0.26 correspond to "low intermediate pairing". In the group of "middle intermediate pairing" the plants from 0.28 to 0.56 are included and in the "high intermediate pairing" the plants from 0.64 to 0.80 . The values from 1.02 to 2.56 are included in the "high pairing group". 
Table 3(b) shows the comparison between an estimation of grouped variance in HCSE, HCSA, HCSJ with regard to HCSV hybrid for both bivalents and paired chromosome complement by the " $F$ " test. In both cases the differences are very significant.

\section{Discussion}

The analysed hybrids have $2 n=28$ chromosomes implicating the $\mathrm{A}, \mathrm{B}$ and $\mathrm{D}$ genomes of wheat and the $\mathrm{R}$ genome of rye. In all hybrids the wheat complement is the same, but the rye genome differs according to hybrid type. Each genome is present in single dose.

Levels of meiotic pairing in T. aestivum are generally low (Riley and Law, 1965; Miller and Riley, 1972; Miller and Chapman, 1972). Dvorak (1977) observed a mean of Xta per cell in eight haploids of Chinese Spring ranging from $0 \cdot 17$ to $0 \cdot 35$. However, there are haploids from other cultivars of T. aestivum such as Holdfast (Riley, 1960), in which the pairing level is higher than Chinese Spring.

In our study, a large range of variation in meiotic pairing of HCSE and HCSA hybrids was found (table 1). This variation is produced by the presence of some plants with lower pairing levels than those of $T$. aestivum haploids; others with a bivalent mean similar to the haploids; and some with a higher pairing level. The plants of lowest pairing type are found in HCSE hybrids and those with the highest in HCSA hybrids. The meiotic behaviour of four HCSV hybrids analysed (table 1) indicates that all bivalent means are similar to those of the wheat haploids, with little variation in range.

There are differences between the average means of bivalents in the different types of hybrids. The HCSE hybrids have a bivalent mean of 0.27 ; this value is similar to the one obtained in wheat-rye hybrids when chromosome $5 \mathrm{~A}$ is lacking (Romero and Lacadena, 1980). This chromosome carries promotor genes of homoeologous pairing. This bivalent mean is the lowest of all the analyzed hybrids, since they have a great number of plants with the lowest pairing levels (fig. 1). The bivalent mean of HCSA hybrids is $0 \cdot 44$. In these hybrids, the maximum value of bivalents per cell was found, and plants in the lowest pairing levels were lacking (fig. 1). The HCSJ hybrids behaved in a similar manner to the HCSA ones, the bivalent mean being very similar. There were no plants in the lowest levels of pairing but in this case the promotion levels in the HCSA hybrids were never reached (Romero and Lacadena, 1982). The bivalent mean of HCSV hybrids is similar to that of HCSA hybrids. However, their behaviour is different because the plants are grouped around the bivalent mean (fig. 1).

There are no significant differences between bivalent and Xta per cell distributions in any of the hybrid types. These results agree with those of Lelley (1976), Viegas (1980) and Romero and Lacadena (1982). In this respect our data demonstrates that the rye effect would be exercised on the number of paired chromosomes and not on the chiasmata per cell. Although the bivalent distributions of all hybrids are similar, the $\chi^{2}$ test demonstrates significant differences in all cases in which HCSV hybrids are not implicated in the comparison (table 2). For the most frequent classes $(0,1,2$ bivalents), the distribution of the last hybrids is situated in the middle of the others. This fact might explain the absence of significant differences. 
According to the variation ranges found in homoeologous pairing for each hybrid type, the variances observed are higher for HCSE, HCSA and HCSJ hybrids than for HCSV plants (table 3).

All the results obtained for the four types of hybrids are summarised in fig. 1. First of all we will analyse the pairing levels independently of the hybrid type. Plants with a bivalent mean lower than that of $T$. aestivum haploids are included in the level of "low pairing". The bivalent mean of these plants is similar to those of wheat-rye hybrids in which the $3 \mathrm{~B}$ chromosome is absent (Romero and Lacadena, 1980). This chromosome carries genes promoting homoeologous pairing. Genetic combinations of rye with suppressor activity for homoeologous pairing might exist in these hybrids. Lelley (1976) suggest the existence of rye promotor and suppressor genes of homoeologous pairing. In this sense, Romero and Lacadena (1980) found a promotor or suppressor effect of rye B-chromosomes depending upon the wheat genetic background.

The plants included in the level of "low intermediate" and "middle intermediate pairing" may have genotypes of rye without an influence upon homoeologous pairing since their bivalent mean is similar to wheat haploids.

Nevertheless, the extreme values of the two levels could be explained by a slight rye promotor and suppressor effect since the considered range is larger than that found in most of the wheat haploids.

In the group of "high intermediate pairing", the bivalent mean is higher than that of wheat haploids. In this case the rye genes promote the association of homoeologous chromosomes.

A higher promotor effect is detected in the plants of the "high pairing" group. Lelley (1976) pointed out that the genetic combinations promoting very high pairing would be exceptional. This might explain the low number of our plants included in these levels. On the other hand, the data obtained in the homogeneity studies suggests that in each group the rye affects the pairing in a similar way. In those cases where the $\chi^{2}$ test is not available (plants of "low pairing" group and HCSV hybrids), the bivalent mean is similar. In conclusion, our results indicate that the rye genes can promote, suppress or may not affect the homoeologous pairing, depending upon the particular genotype of rye present in the hybrids.

According to the meiotic behaviour of different hybrids, each rye type has a particular effect on the homoeologous pairing. In the case of hybrids with the cultivar Elbon, there are plants included in the "low pairing" group and genetic combinations without effects on the homoeologous pairing and even others promoting it.

HCSA hybrids have the highest bivalent mean (2.56). Dvorak (1977) analysed wheat-rye hybrids (cv. Prolific) observing that the highest Xta per cell mean was $3 \cdot 59$. This value is similar to the maximum value of pairing occurring in our HCSA hybrids (the bivalent mean, 2.56, corresponds to 3.32 Xta per cell). On the basis of this data the promotor effect of cv. Ailés is comparable to that of cv. Prolific. Moreover, this value is similar to the one observed in wheat-rye hybrids where the $3 \mathrm{D}$ chromosome, which contains suppressor genes for homoeologous pairing, is absent (Romero and Lacadena, 1980). This agrees with the idea that promotor genes of homoeologous pairing are present in the cv. Ailés. In this cultivar, a suppressor effect as important as that found in the cv. Elbon, has not been 
demonstrated because the lowest pairing level of HCSA hybrids is situated in the wheat haploid range.

The average bivalent mean of $\mathrm{HCSJ}$ is $0 \cdot 43$. In comparison to the other types of hybrids there is a proportional increase of the number of plants included in "high intermediate pairing" level.

All HCSV hybrids have bivalent means similar to those of wheat haploids indicating that, $S$. vavilovii has practically no effect on the homoeologous pairing control of wheat.

The meiotic pairing variation range is extensive in the HCSE, HCSA and HCSJ hybrids (fig. 1). This fact is related to the type of reproduction of Elbon, Ailés and JNK cultivars. These three cultivars are allogamous with a high level of heterozygosity. Therefore, many different genetic combinations, with different effects on the homoeologous pairing control of wheat, would exist. Since the rye species used in HCSV hybrids is autogamous, all plants would have the same genetic combinations and therefore similar effects upon the wheat. This fact could explain the slight variation range of these hybrids. Thus, the differences in the variation range (see table 3 ) cannot be attributed to chance, but to the reproductive mechanism (allogamy and autogamy) of the different rye cultivars and species used.

\section{REFERENCES}

DOVER, G. A. AND, RILEY, R. 1972. Variation at two loci affecting homoeologous meiotic chromosome pairing in Triticum aestivum $\times$ Aegilops mutica hybrids. Nature, New Biol., $235,61-62$.

DVORAK, J. 1972. Genetic variability in Aegilops speltoides affecting homoeologous pairing in wheat. Can. J. Genet. Cytol., 14, 371-380.

DVORAK, J. 1977. Effect of rye on homoeologous chromosome pairing in wheat $\times$ rye hybrids. Can. J. Genet. Cytol., 19, 549-556.

KALTSIKES, P. J. 1974. Methods for Triticale production. Z. Pflan., 71, 264-286.

LELLEY, T. 1976. Induction of homoeologous pairing in wheat by genes of rye suppressing chromosome 5B effect. Can. J. Genet. Cytol., 18, 485-489.

MELlO-SAMPAYO, T. 1971. Promotion of homoeologous pairing in hybrids of Triticum aestivum $\times$ Aegilops longissima. Genet. Iber., 23, 1-9.

MILLER, T. E. AND CHAPMAN, V. 1976. Aneuhaploids in bread wheat. Genet. Res., 28, 37-45.

MILLER, T. E. AND RILEY, R. 1972. Meiotic chromosome pairing in wheat rye combinations. Genet. Iber., 24, 241-250.

NARANJO, T., LACADENA, J. R. AND GIRALDEZ, R. 1979. Interaction between wheat and rye genomes on homologous and homoeologous pairing. Z. Pflan., 82, 289-305.

RILEY, R. 1960. The diploidisation of polyploid wheat. Heredity, 15, 407-429.

RILEY, R. AND LAW, C. N. 1965. Genetic variation in chromosome pairing. Adv. Genet., 13, 57-114.

ROMERO, C. AND LACADENA, J, R. 1980. Interaction between rye B-chromosomes and wheat genetic systems controlling homoeologous pairing. Chromosoma, 80, 33-48.

ROMERO, C. AND LACADENA, J. R. 1982. Effect of rye B-chromosomes on pairing in Triticum aestivum $\times$ Secale cereale hybrids. Z. Pflan., 89, 39-46.

ROOTHAAN, M. AND SYBENGA, J. 1976. No 5B-compensation by rye B-chromosomes. Theor. Appl. Genet., 48, 63-66.

UPADHYA, M. D. AND SWAMINATHAN, M. S. 1967. Mechanisms regulating chromosome pairing in Triticum. Biol. Zbl., 86, 239-255.

VIEGAS, W. S. 1980. The effect of B-chromosomes of rye on the chromosome association in $F_{1}$ hybrids Triticum aestivum $\times$ Secale cereale in absence of chromosome $5 B$ or 5D. Theor. Appl. Genet., 56, 193-198.

WALL, A. M., RILEY, R. AND CHAPMAN, V. 1971. Wheat mutants permitting homoeologous meiotic chromosome pairing. Genet. Res., 18, 311-328. 